\title{
Heat-shock protein 60 of Porphyromonas gingivalis may induce dysfunction of human umbilical endothelial cells via regulation of endothelial-nitric oxide synthase and vascular endothelial-cadherin
}

\author{
CUNJIN WU ${ }^{1}$, SHIJIE GUO ${ }^{1}$, YUANJIE NIU ${ }^{2}$, LIMIN YANG ${ }^{1}$, \\ BAINIAN LIU ${ }^{1}$, NING JIANG ${ }^{2}$, MING SU $^{3}$ and LIN WANG ${ }^{1}$ \\ ${ }^{1}$ Department of Geratology, The Second Hospital of Tianjin Medical University; ${ }^{2}$ Department of Urology, \\ Tianjin Institute of Urology, The Second Hospital of Tianjin Medical University, Tianjin 300211; \\ ${ }^{3}$ State Key Laboratory of Cardiovascular Disease, Fuwai Hospital, National Center for Cardiovascular Diseases, \\ Chinese Academy of Medical Sciences and Peking Union Medical College, Beijing 100037, P.R. China
}

Received February 3, 2016; Accepted April 27, 2016

DOI: 10.3892/br.2016.693

\begin{abstract}
Accumulating evidence has established that periodontitis was an independent risk factor for coronary heart disease (CAD). Porphyromonus gingivalis ( $P$. gingivalis), a major periodontal pathogen, has already been shown to have a significant role in the inflammatory response of CAD in vivo. The aim of the present study was to identify whether $P$. gingivalis heat-shock protein 60 (HSP60) induced the dysfunction of human umbilical vein endothelial cells (HUVECs) in vitro. HUVECs were stimulated with a range of $P$. gingivalis HSP60 concentrations $(1,10$ and $100 \mathrm{ng} / \mathrm{l})$ at different time-points. The levels of vascular endothelial (VE)-cadherin, endothelial nitric oxide synthase (eNOS) and cysteinyl aspartate-specific protease-3 (caspase-3) were measured using western blot analysis. The apoptotic rate of HUVECs was detected using flow cytometry. $P$. gingivalis HSP60 at a concentration of $10 \mathrm{ng} / \mathrm{l}$ significantly decreased the expression levels of $\mathrm{VE}$-cadherin and eNOS protein at $24 \mathrm{~h}$ stimulation, whereas no difference in these proteins was identified following a low dose of $P$. gingivalis HSP60 (1 ng/l). $P$. gingivalis HSP60 at $100 \mathrm{ng} / \mathrm{l}$ significantly downregulated the expression levels of VE-cadherin and eNOS protein at $12 \mathrm{~h}$ in HUVECs. However, the cleavage of caspase-3 showed an opposing change at different concentrations. Consistently, $P$. gingivalis HSP60 induced apoptosis of HUVECs in a concentration-dependent
\end{abstract}

Correspondence to: Dr Lin Wang, Department of Geratology, The Second Hospital of Tianjin Medical University, 23 Pingjiang Road, Tianjin 300211, P.R. China

E-mail: wang.lin@medmail.com.cn

Key words: Porphyromonas gingivalis, heat-shock protein, E-cadherin, endothelial nitric oxide synthase, apoptosis manner. These results indicated that $P$. gingivalis HSP60 may induce dysfunction and apoptosis in HUVECs via downregulating the expression levels of VE-cadherin and eNOS, and promoting the cleavage of caspase-3.

\section{Introduction}

Previous studies indicated that periodontitis may be associated with a higher risk of coronary heart disease (CAD) (1-4), independent of established cardiovascular risk factors. Periodontitis is a chronic tissue-destruction inflammatory state that is predominantly induced by Porphyromonus gingivalis ( $P$. gingivalis) in the gingival pockets of certain individuals with advanced and severe periodontal disease.

$P$. gingivalis may promote transient bacteremia during tooth brushing, chewing or dental procedures (5-7). Certain studies have identified that $P$. gingivalis was detected frequently in atheromatous plaques of the aorta and coronary artery, and it was reported to perpetuate systemic inflammation (8-10). Additionally, $P$. gingivalis induces macrophage foam cell formation (11) and stimulates oxidation of low-density lipoprotein (12). Certain studies show that $P$. gingivalis lipopolysaccharide (LPS) could induce the expression of intercellular adhesion molecule 1 and vascular cell adhesion molecule 1 in human umbilical vein endothelial cells (HUVECs) $(13,14)$, which significantly enhances trans-endothelial migration of inflammatory cells.

Furthermore, atherosclerosis can be triggered and aggravated by the pathogen-driven antigenic peptide from $P$. gingivalis heat-shock protein 60 (HSP60) (15-17). An overall $55 \%$ homology exists between human and bacterial HSP60 that canevenreach $72 \%$ atcertaindomains of the 573 -amino-acid-long molecule (18). P. gingivalis HSP60 is reported to accelerate the development of experimental atherosclerosis by cross-reactivity of the immune response to bacterial HSPs (19). However, Jeong et al (20) found that $P$. gingivalis HSP60 peptides have distinct roles in the development of atherosclerosis; 
peptide 14 or 19 from $P$. gingivalis HSP60 may have either an anti- or pro-atherogenic role, respectively, in the ApoE(-/-) mouse model of infection-triggered atherosclerosis through distinct mechanisms operating in the polarization of $\mathrm{T}$ cells. Additionally, in a clinical study, a strong positive correlation was found between high levels of soluble HSP60 and the risk of CAD (21). Soluble HSP60 levels directly correlate with the presence of classic risk factors of atherosclerosis, such as elevated low-density lipid cholesterol levels, and with particular proinflam $\neg$ matory markers, such as tumor necrosis factor- $\alpha$ (22).

However, the potential pathways linking periodontitis and cardiovascular disease remain to be elucidated (23-25) and the underlying molecular mechanisms from $P$. gingivalis HSP60 regarding the association between periodontitis and atherosclerosis require further investigation. In the present study, the aim was to investigate whether $P$. gingivalis HSP60 treatment leads to the dysfunction of HUVECs directly by affecting the protein expression levels of endothelial nitric oxide synthase (eNOS) and vascular endothelial (VE)-cadherin.

\section{Materials and methods}

Cell culture. HUVECs were kindly provided as a gift by Dr Yun Mu (Tianjin Medical University, Tianjin, China). Cell culture media and supplements were purchased from Gibco (Thermo Fisher Scientific, Inc., Waltham, MA, USA). Fetal bovine serum (FBS) was purchased from Gibco (Thermo Fisher Scientific, Inc.). P. gingivalis HSP60 was purchased from Hongling Longcheng Technology Co., Ltd. (Beijing, China). The cells were cultured in RPMI-1640 medium supplemented with $10 \% \mathrm{FBS}$ at $37^{\circ} \mathrm{C}$ in a humidified incubator with $5 \% \mathrm{CO}_{2}$. The culture medium was exchanged every $48 \mathrm{~h}$. HUVECs up to passage 6 were used for the experiments.

Cell viability. Cell viability was determined using the MTT assay. HUVECs were seeded in 96-well culture plates at a density of $0.5 \times 10^{4}$ cells/well and incubated overnight at $37^{\circ} \mathrm{C}$. Following treatment with $P$. gingivalis HSP60 at different concentrations (1, 10 and $100 \mathrm{ng} / \mathrm{l})$, cells were incubated with $5 \mathrm{mg} / \mathrm{ml} \mathrm{MTT}$ for $24 \mathrm{~h}$. Subsequently, the MTT-containing growth medium was replaced with $100 \mu$ l of dimethyl sulfoxide (DMSO) and mixed thoroughly for $10 \mathrm{~min}$. The optical density readings of each well were determined at $570 \mathrm{~nm}$ using a microplate reader (ELx808; BioTek Instruments, Inc., Winooski, VT, USA). The effect of $P$. gingivalis HSP60 on cell viabilities was expressed as the percentage of viable cells in the treated groups compared to the DMSO control. Values [mean \pm standard deviation (SD)] are from three independent experiments.

Sodium dodecyl sulfate-polyacrylamide gel electrophoresis (SDS-PAGE) and western blot analysis. The cell layer was washed with $3 \mathrm{ml}$ of phosphate-buffered saline (PBS) twice. Following treatment (1, 10 and $100 \mathrm{ng} / \mathrm{l} P$. gingivalis HSP60 for $2,6,12$ or $24 \mathrm{~h}$ ), the cells were homogenized in an ice bath using sonification ( 3 times for $15 \mathrm{sec}, 50 \mathrm{~Hz}$ ) with $1 \mathrm{ml}$ of radioimmunoprecipitation assay lysis buffer containing $400 \mu \mathrm{l}$ of protease inhibitors of phenylmethylsulfonyl fluoride (Thermo Fisher Scientific, Inc.). The homogenate was

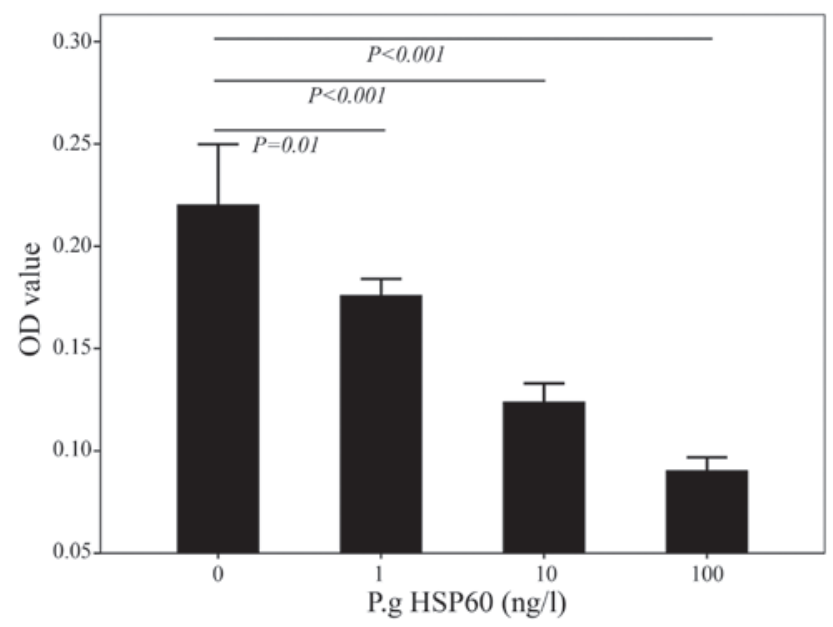

Figure 1. Viabilities of HUVECs following P. gingivalis HSP60 treatment at various concentrations for $24 \mathrm{~h}$. Data are expressed as \% viability to that of the controls. Values are mean \pm standard deviation, $\mathrm{n}=3$. Compared to $1 \mathrm{ng} / \mathrm{l}$ $P$. gingivalis HSP60-treated HUVECs, $\mathrm{P}=0.004$ for $10 \mathrm{ng} / 1$ and $\mathrm{P}<0.001$ for $100 \mathrm{ng} / \mathrm{l}$. Compared to $10 \mathrm{ng} / 1$ P. gingivalis HSP60-treated HUVECs, $\mathrm{P}=0.035$ for $100 \mathrm{ng} / \mathrm{l}$. OD, optical density; P. g, Porphyromonus gingivalis; HSP60, heat-shock protein 60; HUVECs, human umbilical endothelial cells.

collected and centrifuged at 12,000 x g for $15 \mathrm{~min}$, and the supernatant was used as a lysate for further determinations. Protein concentration was determined by the $\mathrm{BCA}^{\mathrm{TM}}$ protein assay kit (Thermo Fisher Scientific, Inc.) according to the manufacturer's protocol. Western blot analysis was performed as described previously (26). Equal amounts of cellular protein $(30 \mu \mathrm{g})$ underwent electrophoresis on a gradient SDS-PAGE (4-10\% gel) and the samples were electrotransferred onto nitrocellulose membranes in a buffer consisting of $25 \mathrm{mM}$ Tris, $192 \mathrm{mM}$ glycine and $20 \%$ methanol ( $\mathrm{pH} \mathrm{8.4)} \mathrm{for} 2 \mathrm{~h}$ at a constant voltage $(100 \mathrm{~V})$ with cooling. The following primary antibodies were used for blotting: $\beta$-actin (cat. no. M20010; monoclonal mouse anti-human; 1:500; Abmart, Inc., Berkeley Heights, NJ, USA), VE-cadherin (cat. no. SAB1306131; polyclonal rabbit anti-human; 1:500; Sigma-Aldrich, St. Louis, MO, USA), eNOS (cat. no. SAB4502013; polyclonal rabbit anti-human; 1:5,000; Sigma-Aldrich), caspase-3 (cat. no. C9598; polyclonal rabbit anti-human; 1:500; Sigma-Aldrich) and cleaved caspase-3 (cat. no. SAB4503294; polyclonal rabbit anti-human; 1:500; Sigma-Aldrich). The secondary antibodies include IRDye (1:3,000; Abmart, Inc.). Immunocomplexes were detected using the ECL western blotting detection kit (GenMed, Inc., Houston, TX, USA). All the other reagents were purchased from Sigma-Aldrich. The specific proteins were visualized by an Odyssey ${ }^{\mathrm{TM}}$ infrared imaging system (LI-COR, Inc., Lincoln, NE, USA).

Flow cytometry analysis for apoptosis quantification. Following the designated treatment $(1,10$ and $100 \mathrm{ng} / \mathrm{l} \mathrm{P}$. gingivalis HSP60), annexin V-fluorescein isothiocyanate-conjugated (FITC)/propidium iodide (PI) apoptosis detection kit (Invitrogen, Thermo Fisher Scientific, Inc.) was used according to the manufacturer's protocol. In brief, the cells were centrifuged at $300 \mathrm{x}$ g for $5 \mathrm{~min}$, washed with cold PBS, and resuspended in $100 \mu \mathrm{l}$ of binding buffer. Annexin V-FITC (5 $\mu \mathrm{l})$ and PI $(5 \mu \mathrm{l})$ were added to each sample, and the mixture was incubated 
A

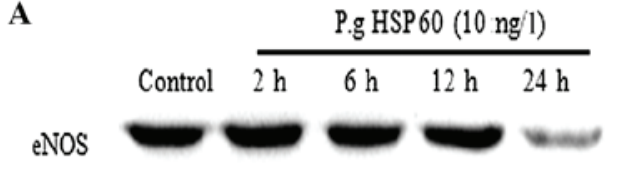

$\beta$-actin

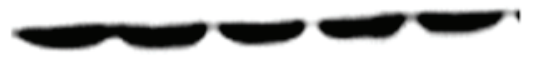

C

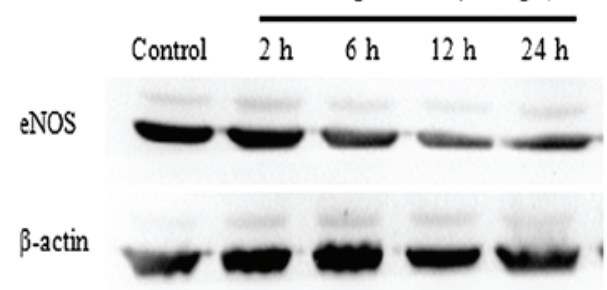

B

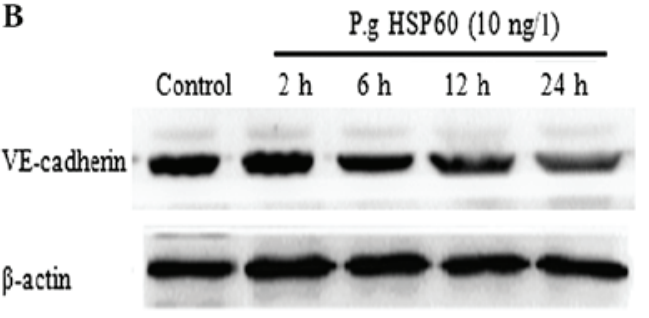

D P.g HSP60 (100 ng/1)

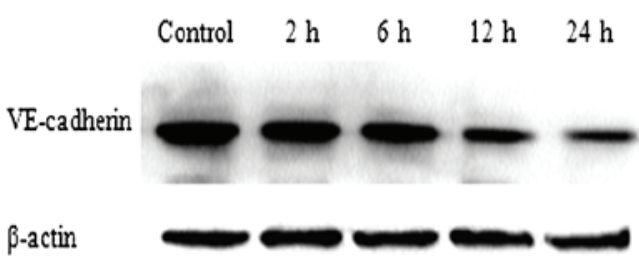

Figure 2. Effects of $P$. gingivalis HSP60 on the protein expression levels of eNOS and VE-cadherin at different time-points. The HUVECs were cultured with $P$. gingivalis HSP60 stimulation (10 and $100 \mathrm{ng} / \mathrm{l})$ at different time-points (2, 6, 12 and $24 \mathrm{~h})$. Effects of $P$. gingivalis HSP60 (10 ng/l) on protein expression levels of (A) eNOS and (B) VE-cadherin at different time-points. Effects of $P$. gingivalis HSP60 (100 ng/l) on protein expression levels of (C) eNOS and (D) VE-cadherin at different time-points. P.g, Porphyromonus gingivalis; HSP60, heat-shock protein 60; eNOS, endothelial nitric oxide synthase; VE, vascular endothelial; HUVECs, human umbilical endothelial cells.

at $4^{\circ} \mathrm{C}$ in the dark for $15 \mathrm{~min}$. The cells were immediately subjected to fluorescence-activated cell sorting analysis (BD Accuri C6; BD Biosciences, San Jose, CA, USA) within 1 h. For cells in the early apoptotic stage, membrane phosphatidylserine was exposed and combined with annexin V. The cells were stained with annexin $\mathrm{V}$ with no PI fluorescence and recorded as annexin V (+)/PI (-). The membranes of dead cells and cells in the late apoptotic stage were permeable to PI. These cells were stained with annexin $\mathrm{V}$ and $\mathrm{PI}$ and were recorded as annexin $\mathrm{V}$ $(+) /$ PI (+). Finally, annexin V (+)/PI (-) and annexin V (+)/PI (+) cells were detected under flow cytometry, and the percentages of the total number of cells in each group were compared.

Statistical analysis. Statistical analyses were performed with SPSS 17.0 software (SPSS, Inc., Chicago, IL, USA). Results are provided as mean \pm SD. One-way or two-way analysis of variance tests were applied to compare the different groups. $\mathrm{P}<0.05$ was considered to indicate a statistically significant difference.

\section{Results}

$P$. gingivalis HSP60 inhibits the proliferation of HUVECs. To examine the effects of $P$. gingivalis HSP60 on HUVECs, the HUVECs were first treated with different concentrations of $P$. gingivalis HSP60, and the cell viability was detected using the MTT assay. $P$. gingivalis HSP60 at 1, 10 and $100 \mathrm{ng} / \mathrm{l}$ significantly altered the viability of HUVECs $(\mathrm{P}<0.05)$ (Fig. 1).

$P$. gingivalis HSP60 downregulates the expression levels of eNOS and V-cadherin in HUVECs. HUVECs were incubated with $P$. gingivalis HSP60 (1, 10 and $100 \mathrm{ng} / \mathrm{l})$ at different time-points $(2,6,12$ and $24 \mathrm{~h})$ and the levels of VE-cadherin and eNOS were detected by western blot analysis. The expression levels of VE-cadherin and eNOS proteins were comparable in HUVECs treated with $1 \mathrm{ng} / \mathrm{l}$ of $P$. gingivalis HSP60. The protein expression levels of eNOS at $24 \mathrm{~h}$ following treatment with $P$. gingivalis HSP60 (10 ng/l) were significantly decreased as compared with those at 2, 6 and $12 \mathrm{~h}$ (Fig. 2A), and the protein expression level of VE-cadherin had an opposing effect (Fig. 2B). Additionally, HUVECs treated with P. gingivalis HSP60 (100 ng/l) exhibited a significantly lower eNOS protein expression level following $12 \mathrm{~h}$ of treatment in contrast to the levels at 2 and $6 \mathrm{~h}$ (Fig. 2C), and the protein expression of VE-cadherin was significantly decreased after $12 \mathrm{~h}$ (Fig. 2D). Taken together, these results provide evidence that $P$. gingivalis HSP60 may lead to endothelial dysfunction by the regulation of VE-cadherin and eNOS protein expression levels.

P. gingivalis HSP60 upregulates the expression of caspase-3 and induces apoptosis of HUVECs in a concentration-dependent manner. Caspase-3 was detected by western blot analysis. The expression of the cleavage of caspase- 3 at $24 \mathrm{~h}$ was significantly increased as compared with that of 2, 6 and $12 \mathrm{~h}$ treatment with $P$. gingivalis HSP60 (10 ng/l) (Fig. 3A), and when HUVECs were treated with $P$. gingivalis HSP60 at $100 \mathrm{ng} / 1$, the expression of the cleavage of caspase- 3 at $12 \mathrm{~h}$ was significantly increased as compared with that at 2 and $6 \mathrm{~h}$ (Fig. 3B). The apoptosis of HUVECs was analyzed by annexin V/FITC staining, as shown in Fig. 3C and D. The percentage of total apoptotic cells was $3.86 \pm 0.60,5.52 \pm 0.82$, $22.99 \pm 2.28$ and $27.11 \pm 3.87 \%$ in cells untreated and cells treated with 1,10 and $100 \mathrm{ng} / \mathrm{l}$ of $P$. gingivalis HSP60, respectively. These results showed that $P$. gingivalis HSP60 $(1,10$ and $100 \mathrm{ng} / \mathrm{l})$ stimulation for $24 \mathrm{~h}$ significantly increased the apoptotic rate and induced significant apoptosis on HUVECs in a concentration-dependent manner.

\section{Discussion}

There is increasing evidence that $P$. gingivalis has a key role in contributing to the progression of atherosclerosis (8-11). According to a recent study, LPS of $P$. gingivalis may induce 
A

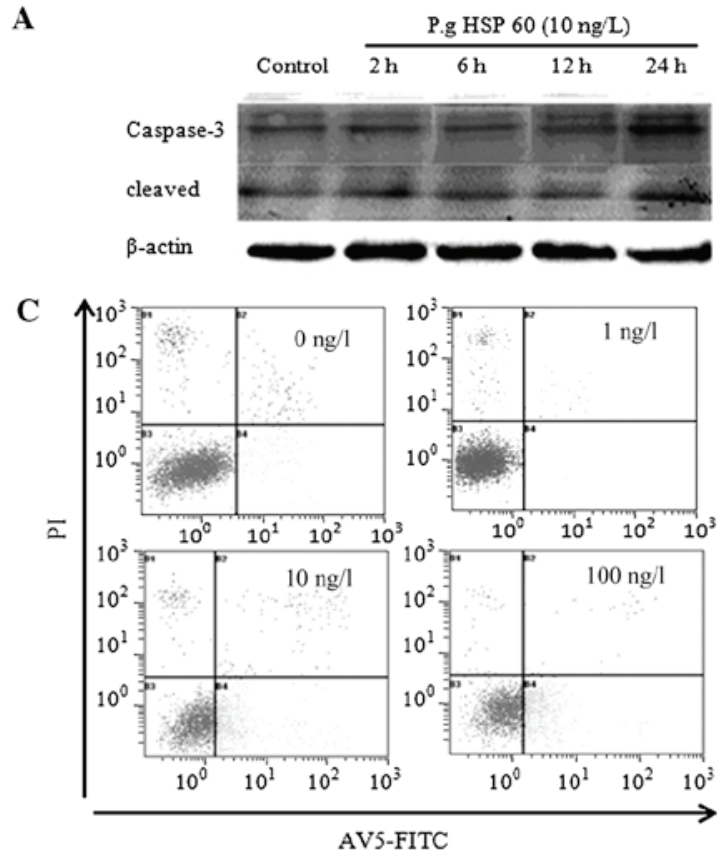

B

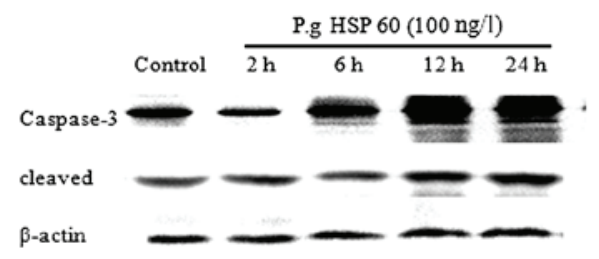

D

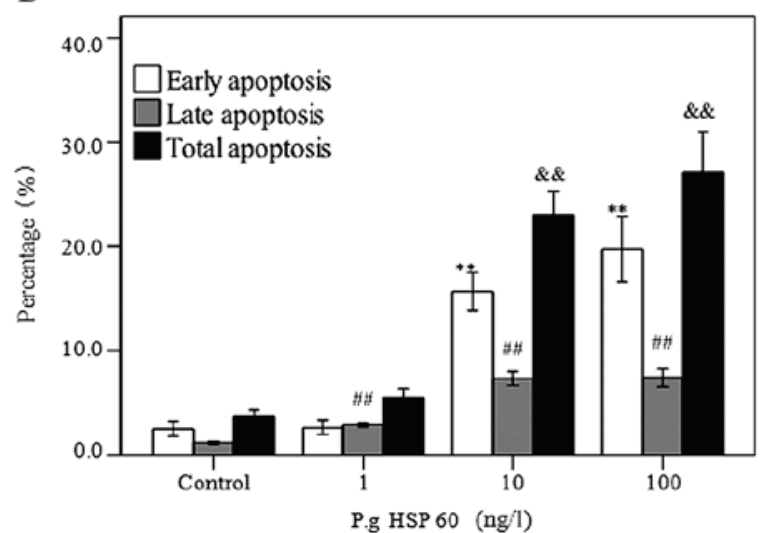

Figure 3. Effects of $P$. gingivalis HSP60 on the protein expression of cleaved caspase-3 protein and apoptosis of HUVECs induced by $P$. gingivalis HSP60. The HUVECs were cultured with $P$. gingivalis HSP60 stimulation (10 and $100 \mathrm{ng} / \mathrm{l})$ at different time-points (2, 6, 12 and 24 h). Effects of $P$. gingivalis HSP60 (10 and $100 \mathrm{ng} / \mathrm{l}$ ) on protein expression of (A and B) cleaved caspase-3 at different time-points. (C and D) Measurement of apoptotic cells treated with P. gingivalis HSP60 (1, 10 and $100 \mathrm{ng} / \mathrm{l})$ for $24 \mathrm{~h}$ was performed. Data are shown as mean \pm standard deviation of three independent experiments. * $<<0.05$ and ${ }^{* *} \mathrm{P}<0.01$ vs. early apoptosis in the control; ${ }^{\#} \mathrm{P}<0.05$ and ${ }^{\# \#} \mathrm{P}<0.01$ vs. late apoptosis in the control; ${ }^{\&} \mathrm{P}<0.05$ and ${ }^{\& \&} \mathrm{P}<0.01$ vs. total apoptosis in the control. $\mathrm{P}$. $\mathrm{g}$, Porphyromonus gingivalis; HSP60, heat-shock protein 60; HUVECs, human umbilical endothelial cells.

arterial endothelial cell apoptosis and the expression of adhesion molecules in endothelial cells in vitro, which may promote atherogenesis (27). Although the $P$. gingivalis-induced dysfunction of HUVECs, including LPS and the immunological mechanism, are well established, little is known regarding the mechanisms involved in $P$. gingivalis HSP60. The association between $P$. gingivalis HSP60 stimulation and HUVEC dysfunction and associated mechanisms are insufficient and require further analysis. The present study assessed the impact of $P$. gingivalis HSP60 on HUVECs. The results proved that co-culture of HUVECs with $P$. gingivalis HSP60 led to decreased viability of HUVECs, as determined by the MTT assay.

As endothelial dysfunction and apoptosis are vital factors in the progression of atherosclerosis, the associated mechanisms of $P$. gingivalis HSP60 on the induction of HUVECs dysfunction and apoptosis were investigated in the present study. The data showed that $P$. gingivalis HSP60 downregulated the expression level of the eNOS protein, which is able to modulate the biologically active gas production of NO. Endothelium-derived NO has an important physiological role in the regulation of vascular tone, and endothelial cell survival and migration $(28,29)$.

Additionally, similar influences of VE-cadherin expression were observed in $P$. gingivalis HSP60-treated HUVECs. VE-cadherin is localized to the adherens junctions, associating with $\alpha$-catenin, $\beta$-catenin, p120-catenin and plakoglobin via its cytoplasmic domains. It has previously been reported that VE-cadherin, as a major regulator of adherens junctions, in particular has an essential role in the regulation of endothelial cell permeability (30-32), migration and assembly of new blood vessels. Loss of entire VE-cadherin or a lack of its cytoplasmic domain induced endothelial apoptosis and prevented normal vascular development in vivo (33). VE-cadherin is an endothelial cell-specific adhesive molecule for the integrity of endothelial cell contacts (31). VE-cadherin has significant functions in the processes of atherosclerosis (33). Thus far, there have been limited studies regarding VE-cadherin expression when HUVECs were treated with $P$. gingivalis HSP60. Information is limited regarding the mechanisms of $P$. gingivalis HSP60-induced atherosclerosis involving the expression of VE-cadherin. The present data identified that VE-cadherin may be one of the factors leading to endothelial dysfunction.

Additionally, the present results showed that $P$. gingivalis HSP60 induced significant apoptosis of HUVECs in a concentration-dependent manner, as shown by the annexin V-FITC/PI assay. Apoptosis is primarily mediated by the activity of caspases. The extrinsic apoptotic pathway involves binding of specific ligands to membrane-bound death receptors, such as Fas/cluster of differentiation 95, which in turn activates caspase-8, facilitating the subsequent activation of terminal effector caspases, such as caspase-3, -6 and $-7(34,35)$. Thus, caspase-3 is pivotal to the death process. Furthermore, $P$. gingivalis HSP60 was also found to a certain extent to induce HUVECs apoptosis through a mechanism that involved caspase-3 activation. It was proved that apoptosis of VE cells resulted in the loss of endothelial integrity, and was a risk factor of atherosclerosis. Therefore, the present study verified the mechanism of $P$. gingivalis HSP60, which led to atherosclerosis by further accelerating apoptosis in HUVECs.

In conclusion, taken together with the results of other studies, we hypothesize that $P$. gingivalis HSP60 has an essential role in 
the dysfunction of HUVECs via the mechanism of regulating eNOS and VE-cadherin expression levels, as well as apoptosis by activating caspase- 3 in a unique manner. These findings provide new mechanistic insights into the effect of $P$. gingivalis HSP60 on HUVECs and the associated pathogenesis of cardiovascular disease and periodontitis. Further study is required to determine the pathway of the $P$. gingivalis HSP60-induced decreased expression of VE-cadherin and eNOS.

\section{Acknowledgements}

The study was completed in the Department of Urology Institute in Tianjin. The authors thank Dr Yuanjie Niu and Dr Ning Jiang for their skilled technical assistance.

\section{References}

1. Dietrich T, Jimenez M, Krall Kaye EA, Vokonas PS and Garcia RI: Age-dependent associations between chronic periodontitis/edentulism and risk of coronary heart disease. Circulation 117: 1668-1674, 2008.

2. Bahekar AA, Singh S, Saha S, Molnar J and Arora R: The prevalence and incidence of coronary heart disease is significantly increased in periodontitis: A meta-analysis. Am Heart J 154: 830-837, 2007

3. Dietrich T, Sharma P, Walter C, Weston P and Beck J: The epidemiological evidence behind the association between periodontitis and incident atherosclerotic cardiovascular disease. J Periodontol 84 (Suppl 4): S70-S84, 2013.

4. Tonetti MS and Van Dyke TE; working group 1 of the joint EFP/AAP workshop: Periodontitis and atherosclerotic cardiovascular disease: Consensus report of the Joint EFP/AAP Workshop on Periodontitis and Systemic Diseases. J Periodontol 84 (Suppl 4): S24-S29, 2013.

5. Forner L, Larsen T, Kilian M and Holmstrup P: Incidence of bacteremia after chewing, tooth brushing and scaling in individuals with periodontal inflammation. J Clin Periodontol 33: 401-407, 2006.

6. Kinane DF, Riggio MP, Walker KF, MacKenzie D and Shearer B: Bacteraemia following periodontal procedures. J Clin Periodontol 32: 708-713, 2005.

7. Guntheroth WG: How important are dental procedures as a cause of infective endocarditis? Am J Cardiol 54: 797-801, 1984.

8. Yumoto H, Chou HH, Takahashi Y, Davey M, Gibson FC III and Genco CA: Sensitization of human aortic endothelial cells to lipopolysaccharide via regulation of Toll-like receptor 4 by bacterial fimbria-dependent invasion. Infect Immun 73: 8050-8059, 2005.

9. Kozarov EV, Dorn BR, Shelburne CE, Dunn WA Jr and Progulske-Fox A: Human atherosclerotic plaque contains viable invasive Actinobacillus actinomycetemcomitans and Porphyromonas gingivalis. Arterioscler Thromb Vasc Biol 25: e17-e18, 2005.

10. Darveau RP: Periodontitis: A polymicrobial disruption of host homeostasis. Nat Rev Microbiol 8: 481-490, 2010.

11. Miyakawa H, Honma K, Qi M and Kuramitsu HK: Interaction of Porphyromonas gingivalis with low-density lipoproteins: Implications for a role for periodontitis in atherosclerosis. J Periodontal Res 39: 1-9, 2004.

12. Giacona MB, Papapanou PN, Lamster IB, Rong LL, D'Agati VD, Schmidt AM and Lalla E: Porphyromonas gingivalis induces its uptake by human macrophages and promotes foam cell formation in vitro. FEMS Microbiol Lett 241: 95-101, 2004.

13. Khlgatian M, Nassar H, Chou HH, Gibson FC III and Genco CA: Fimbria-dependent activation of cell adhesion molecule expression in Porphyromonas gingivalis-infected endothelial cells. Infect Immun 70: 257-267, 2002.

14. Nakamura N, Yoshida M, Umeda M, Huang Y, Kitajima S, Inoue Y, Ishikawa I and Iwai T: Extended exposure of lipopolysaccharide fraction from Porphyromonas gingivalis facilitates mononuclear cell adhesion to vascular endothelium via Toll-like receptor-2 dependent mechanism. Atherosclerosis 196: 59-67, 2008.

15. Jeong E, Lee JY, Kim SJ and Choi J: Predominant immunoreactivity of Porphyromonas gingivalis heat shock protein in autoimmune diseases. J Periodontal Res 47: 811-816, 2012.
16. Rajaiah R and Moudgil KD: Heat-shock proteins can promote as well as regulate autoimmunity. Autoimmun Rev 8: 388-393, 2009.

17. van Puijvelde GH, van Es T, van Wanrooij EJ, Habets KL, de Vos P, van der Zee R, van Eden W, van Berkel TJ and Kuiper J: Induction of oral tolerance to HSP60 or an HSP60-peptide activates $\mathrm{T}$ cell regulation and reduces atherosclerosis. Arterioscler Thromb Vasc Biol 27: 2677-2683, 2007.

18. Craig EA, Gambill BD and Nelson RJ: Heat shock proteins: Molecular chaperones of protein biogenesis. Microbiol Rev 57: 402-414, 1993.

19. Ford PJ, Gemmell E, Hamlet SM, Hasan A, Walker PJ, West MJ, Cullinan MP and Seymour GJ: Cross-reactivity of GroEL antibodies with human heat shock protein 60 and quantification of pathogens in atherosclerosis. Oral Microbiol Immunol 20: 296-302, 2005

20. Jeong E, Kim K, Kim JH, Cha GS, Kim SJ, Kang HS and Choi J: Porphyromonas gingivalis HSP60 peptides have distinct roles in the development of atherosclerosis. Mol Immunol 63: 489-496, 2015.

21. Zhang X, He M, Cheng L, Chen Y, Zhou L, Zeng H, Pockley AG, $\mathrm{Hu} \mathrm{FB}$ and Wu T: Elevated heat shock protein 60 levels are associated with higher risk of coronary heart disease in Chinese. Circulation 118: 2687-2693, 2008.

22. Lewthwaite J, Owen N, Coates A, Henderson B and Steptoe A: Circulating human heat shock protein 60 in the plasma of British civil servants: Relationship to physiological and psychosocial stress. Circulation 106: 196-201, 2002.

23. Van Dyke TE and van Winkelhoff AJ: Infection and inflammatory mechanisms. J Clin Periodontol 40 (Suppl 14): S1-S7, 2013.

24. Schenkein HA and Loos BG: Inflammatory mechanisms linking periodontal diseases to cardiovascular diseases. J Periodontol 84 (Suppl 4): S51-S69, 2013.

25. Kebschull M, Demmer RT and Papapanou PN: 'Gum bug, leave my heart alone!' - epidemiologic and mechanistic evidence linking periodontal infections and atherosclerosis. J Dent Res 89: 879-902, 2010.

26. Kajiyama H, Kikkawa F, Khin E, Shibata K, Ino K and Mizutani S: Dipeptidyl peptidase IV overexpression induces up-regulation of E-cadherin and tissue inhibitors of matrix metalloproteinases, resulting in decreased invasive potential in ovarian carcinoma cells. Cancer Res 63: 2278-2283, 2003.

27. Andrukhov O, Steiner I, Liu S, Bantleon HP, Moritz A and Rausch-Fan X: Different effects of Porphyromonas gingivalis lipopolysaccharide and TLR 2 agonist Pam3CSK4 on the adhesion molecules expression in endothelial cells. Odontology 103: 19-26, 2015.

28. Hobbs AJ, Higgs A and Moncada S: Inhibition of nitric oxide synthase as a potential therapeutic target. Annu Rev Pharmacol Toxicol 39: 191-220, 1999.

29. Busse R and Mülsch A: Calcium-dependent nitric oxide synthesis in endothelial cytosol is mediated by calmodulin. FEBS Lett 265: 133-136, 1990.

30. Smadja DM, Bièche I, Helley D, Laurendeau I, Simonin G, Muller L, Aiach M and Gaussem P: Increased VEGFR2 expression during human late endothelial progenitor cells expansion enhances in vitro angiogenesis with up-regulation of integrin alpha(6). J Cell Mol Med 11: 1149-1161, 2007.

31. Franco CA, Mericskay M, Parlakian A, Gary-Bobo G, Gao-Li J, Paulin D, Gustafsson E and Li Z: Serum response factor is required for sprouting angiogenesis and vascular integrity. Dev Cell 15: 448-461, 2008.

32. Baluk P, Fuxe J, Hashizume H, Romano T, Lashnits E, Butz S, Vestweber D, Corada M, Molendini C, Dejana E, et al: Functionally specialized junctions between endothelial cells of lymphatic vessels. J Exp Med 204: 2349-2362, 2007.

33. Potter MD, Barbero S and Cheresh DA: Tyrosine phosphorylation of VE-cadherin prevents binding of p120- and beta-catenin and maintains the cellular mesenchymal state. J Biol Chem 280: 31906-31912, 2005.

34. Marsden VS, O'Connor L, O'Reilly LA, Silke J, Metcalf D, Ekert PG, Huang DC, Cecconi F, Kuida K, Tomaselli KJ, et al: Apoptosis initiated by Bcl-2-regulated caspase activation independently of the cytochrome c/Apaf-1/caspase-9 apoptosome. Nature 419: 634-637, 2002.

35. Degterev A, Boyce $M$ and Yuan J: A decade of caspases. Oncogene 22: 8543-8567, 2003. 